\title{
Rivaroxaban-Induced Hypersensitivity Syndrome
}

\author{
Charles-Olivier Chiasson, Arnaud Canneva, François-Olivier Roy, and Maxime Doré
}

\section{INTRODUCTION}

$\mathrm{R}^{\mathrm{i}}$ ivaroxaban is a direct oral anticoagulant that selectively and reversibly inhibits factor $\mathrm{Xa}$ in the common pathway, preventing thrombin activation and thus clot formation. ${ }^{1}$ Direct oral anticoagulants are used for the prevention of thromboembolism in atrial fibrillation, the prevention of venous thromboembolism in certain populations, and the treatment of venous thromboembolism. ${ }^{2,3}$ Rivaroxaban has once-daily dosing, the dosage must be adjusted according to renal function, and the drug does not require regular monitoring. Rivaroxaban should not be used for patients receiving strong inhibitors or inducers of cytochrome P450 3A4 isozyme and/or P-glycoprotein, such as itraconazole, ritonavir, carbamazepine, rifampin, and St John's wort. ${ }^{4}$ Common adverse effects associated with rivaroxaban are related to the bleeding risks (e.g., ecchymosis, gastrointestinal bleeding, intracranial hemorrhage). Less common adverse events include an increase in serum transaminases, cholestasis, thrombocytopenia, and StevensJohnson syndrome. ${ }^{5}$

Very few cases of cutaneous reactions related to rivaroxaban have been reported. We describe a rivaroxaban-induced hypersensitivity syndrome manifested by a rash and hepatic cytolysis.

\section{CASE REPORT}

A woman in her early 30s, without relevant medical history and no reported allergies, presented to the emergency department with paresthesia and edema of the left upper limb.* A diagnosis of Paget-Schroetter syndrome associated with use of hormonal contraceptive was made.

Paget-Schroetter syndrome, also known as effort thrombosis, is deep vein thrombosis (DVT) of an upper extremity. ${ }^{6}$ It is

\footnotetext{
*The patient provided verbal consent for publication of this case report. In addition, potentially identifying details not pertinent to understanding the case have been omitted.
}

relatively uncommon, accounting for $20 \%$ to $30 \%$ of upperextremity DVT, with upper-extremity DVT accounting for only $4 \%$ to $10 \%$ of all cases of DVT. ${ }^{6}$ Pathogenic factors for initiation or progression of Paget-Schroetter syndrome are usually anatomic venous abnormality and repetitive endothelial trauma secondary to vigorous and sustained movements of the upper extremity. ${ }^{7}$ Although its pathogenesis is still poorly understood, it is hypothesized to differ from other forms of DVT, which are usually associated with blood stasis or thrombophilic disorders (or both). ${ }^{7}$

In the case presented here, the patient had been taking an oral contraceptive (ethinyl estradiol $20 \mu \mathrm{g}$ and levonorgestrel $100 \mu \mathrm{g}$ ) once daily for more than a year, which was stopped after the diagnosis of Paget-Schroetter syndrome. The patient was not taking any other prescribed medications, over-the-counter medications, or natural health products.

At the time of admission, all laboratory parameters were within normal ranges, including renal function (creatinine $61 \mu \mathrm{mol} / \mathrm{L}$, glomerular filtration rate $>60 \mathrm{~mL} / \mathrm{min}$ ), liver enzymes (aspartate aminotransferase [AST] $17 \mathrm{U} / \mathrm{L}$, alanine aminotransferase $[\mathrm{ALT}]$ not available, total bilirubin $7 \mu \mathrm{mol} / \mathrm{L}$ ), and complete blood count (leukocytes $6.3 \times 10^{9} / \mathrm{L}$, eosinophils $0.1 \times 10^{9} / \mathrm{L}$, hemoglobin $133 \mathrm{~g} / \mathrm{L}$, platelets $\left.193 \times 10^{9} / \mathrm{L}\right)$. Catheter-directed thrombolytic therapy was not considered initially, and anticoagulation with a direct oral anticoagulant was initiated. Rivaroxaban $15 \mathrm{mg}$ orally every $12 \mathrm{~h}$ for 3 weeks, to be followed by $20 \mathrm{mg}$ orally once daily for 3 months, was planned. The patient started anticoagulation immediately and was discharged from the emergency department. However, 3 days later, she returned to the hospital because the edema of her left upper limb had progressed significantly. At that time, she was admitted to receive catheter-directed thrombolytic therapy.

Alteplase $1 \mathrm{mg} / \mathrm{h} \mathrm{IV}$ was administered for a total of $48 \mathrm{~h}$ according to the hospital thrombolysis protocol. Infusion of unfractionated heparin (UFH) was initiated concurrently with 
the alteplase. The UFH was adjusted as per hospital protocol, with a target partial thromboplastin time (PTT) 1.5 to 2 times the upper limit of control PTT. The UFH was administered for the entire hospital stay. After 5 days in hospital and completion of the catheter-directed thrombolytic therapy, the patient was discharged again. She was instructed to continue the rivaroxaban treatment as previously planned.

Three days after this second discharge, the patient presented to the internal medicine outpatient clinic with a mild pruritic papular rash involving only the trunk area. The patient denied other constitutional symptoms such as fever, fatigue, arthralgia, or lymphadenopathy. She also denied having infectious contacts or recent travel. Apart from rivaroxaban, no other medication had been taken in the period between discharge from hospital and onset of the rash, which had appeared 1 day after the patient restarted rivaroxaban. The patient had tried to control the skin rash with 1 dose of diphenhydramine $50 \mathrm{mg}$, which did not alleviate the symptoms. Skin biopsy showed an acute spongiotic dermatitis with perivascular lymphocytes and eosinophilic infiltrates compatible with a drug reaction. Elevation of ALT and AST levels to 209 and $115 \mathrm{U} / \mathrm{L}$, respectively, was noted. The patient also showed slight anemia (hemoglobin $119 \mathrm{~g} / \mathrm{L}$ ) and slight elevation of C-reactive protein $(10.3 \mathrm{mg} / \mathrm{L})$. All other laboratory values were within the normal range, including the absolute eosinophil count $\left(0.4 \times 10^{9} / \mathrm{L}\right)$.

A drug-induced hypersensitivity syndrome (DIHS) due to rivaroxaban was primarily suspected, as no other differential diagnosis was deemed probable. The rivaroxaban was stopped, and the patient was discharged with a prescription of enoxaparin $70 \mathrm{mg}(1 \mathrm{mg} / \mathrm{kg})$ subcutaneously twice daily. No systemic or topical corticosteroids were used.

Six days later, at the follow-up appointment, the patient was feeling well, and the rash had completely resolved. The patient reported that the rash had disappeared within $48 \mathrm{~h}$ after discontinuation of rivaroxaban. AST and ALT levels had declined to 130 and $88 \mathrm{U} / \mathrm{L}$, respectively. The anemia had also resolved (hemoglobin $132 \mathrm{~g} / \mathrm{L}$ ), and the level of C-reactive protein had decreased to $3.0 \mathrm{mg} / \mathrm{L}$. All other laboratory parameters were still within normal range. No particular adverse effects were reported with the use of enoxaparin. Warfarin was initiated, and once the international normalized ratio reached 2 , the enoxaparin was discontinued. Follow-up 1 month later showed that AST and ALT levels were within normal limits $(\leq 40 \mathrm{U} / \mathrm{L})$, as were the values for all other laboratory parameters.

\section{DISCUSSION}

Drug hypersensitivity reactions are known to be idiosyncratic and difficult to predict. Gell and Coombs first classified hypersensitivity reactions, according to a wide variety of clinical manifestations. ${ }^{8}$ Essentially any organ or system can be involved. ${ }^{7}$ Classification of hypersensitivity reactions is also diverse and comprises many specific syndromes with various degrees of severity. ${ }^{9-11}$ In the case presented here, the rapid onset of the rash after a second exposure to rivaroxaban was compatible with a delayed hypersensitivity reaction, which is a T lymphocytemediated reaction. ${ }^{9,10}$

Given the clinical presentation of this case, the most plausible diagnosis was a mild DIHS due to rivaroxaban. A DIHS is similar to the drug rash with eosinophilia and systemic symptoms (DRESS) syndrome, except that eosinophilia is not present. The most common causes of DIHS/DRESS are allopurinol, sulfonamide antibiotics, and aromatic anticonvulsants. ${ }^{12}$ Stevens-Johnson syndrome and toxic epidermal necrolysis are other examples of severe delayed hypersensitivity reactions. Although the patient described here recovered rapidly, it could be hypothesized that her rapid recognition of symptoms and the quick discontinuation of the causative drug prevented progression to a more severe form of DIHS and could also explain the absence of eosinophilia. Internal organ involvement is generally asymptomatic and may not necessarily be recognized. If the patient had pursued rivaroxaban treatment further, the rash might have expanded or worsened, and the hepatic cytolysis might have progressed to a more severe degree. However, elevation of ALT levels to more than 3 times the normal upper limit nonetheless bears witness to the severity of the reaction and indicates systemic involvement in addition to the cutaneous manifestation.

Cutaneous reactions to rivaroxaban, although possible, are relatively uncommon. According to the product monograph, ${ }^{4}$ blisters $(1.4 \%)$, pruritus $(1.8 \%)$, and wound discharge $(2.8 \%)$ have been reported in adult clinical trials. Stevens-Johnson syndrome has also been reported during postmarketing surveillance. ${ }^{4} \mathrm{~A}$ postmarketing report by the US Food and Drug Administration raised concerns about the hepatic safety of direct oral anticoagulants, including rivaroxaban. ${ }^{13}$ We conducted a systematic search using PubMed, Ovid MEDLINE, and Ovid Embase. All papers in French or English that reported a hypersensitivity reaction to rivaroxaban were included, whether or not there was a skin rash. Animal studies were excluded. Seven case reports were identified, ${ }^{14-20}$ of which 4 presented similarities to the present case and were accessible to the authors. ${ }^{17-20}$

Vernon and others ${ }^{17}$ reported a hypersensitivity reaction to rivaroxaban used to treat pulmonary embolism. The presentation in that case differed markedly, with the patient experiencing blisters and a "red, bumpy, itchy rash" all over his body after only 1 dose. Laboratory studies showed no abnormalities, including liver enzymes. Discontinuation of the drug did not alleviate the symptoms. ${ }^{17}$

In another case report, ${ }^{18}$ rivaroxaban at a dose of $10 \mathrm{mg}$ daily was suspected to have caused a hypersensitivity reaction characterized by elevated leukocyte count, neutrophilia, and 
eosinophilia, without any other laboratory abnormalities. The rash was maculopapular in nature, but presented with a few pustules and within only 2 days after initiation of rivaroxaban. Discontinuation of rivaroxaban and supportive treatments led to patient improvement, ${ }^{18}$ as occurred in the case presented here.

Rivaroxaban has also been previously associated with probable cases of DRESS syndrome. ${ }^{19,20}$ In a case reported by Barrett and others, ${ }^{19}$ the rash was similar to that of the current case, but the time to onset was about 6 weeks, and the rash was associated with other symptoms, such as fever, fatigue, and arthralgia, along with hepatic cytolysis. The patient in that case was treated with corticosteroids, which led to a marked decrease in liver enzymes. In the current case, no treatment was given, as discontinuation of rivaroxaban was sufficient to resolve the rash and normalize the results of liver enzyme testing. Similarly, Radu and others ${ }^{20}$ described a case of DRESS syndrome induced by rivaroxaban at a dosage of $10 \mathrm{mg}$ daily. Chills and persistent fever up to $40.5^{\circ} \mathrm{C}$ appeared after 10 days of rivaroxaban treatment, followed by generalized skin erythema with pruritus on day 15 . Because the drug was not stopped until day 20, the patient progressively deteriorated and required admission to an intensive care unit. After stabilization, the patient required a long-term course of corticosteroids, an approach that is often recommended by experts for severe DIHS/DRESS, although supporting literature is lacking. ${ }^{21}$

In addition to these previously reported cases, one report of rivaroxaban-induced serum sickness reaction has been published. ${ }^{22}$ This type of reaction is classified as an immune complex-mediated hypersensitivity (Gell and Coombs type 3 reaction). ${ }^{22}$

In the case reported here, a DIHS due to rivaroxaban seemed to be the only possible explanation for the patient's rash. The favourable outcome after discontinuation of the presumed offending agent is another element favouring the diagnosis of rivaroxaban-induced hypersensitivity syndrome. It seems unlikely that other medications could have caused this reaction, as rivaroxaban was the sole medication taken by the patient after discharge. Furthermore, only 2 other medications, alteplase and UFH, had been taken in the days before onset of the reaction, but neither of these was suspected as a possible causative agent. Alteplase is rapidly eliminated and is not associated with this kind of hypersensitivity reaction. Angioedema and anaphylactoid reactions are the only hypersensitivity reactions to alteplase that have been reported in literature, and they occur only during the drug infusion. ${ }^{23-26}$ Delayed hypersensitivity reactions have been described with subcutaneous UFH. Surprisingly, patients with delayed hypersensitivity reactions to subcutaneous heparins tolerate IV administration of UFH, ${ }^{27}$ which was used in the case reported here. Considering the very short half-life of UFH and the fact that it was stopped more than $72 \mathrm{~h}$ before manifestation of the rash, the plasma concentration of UFH would have been undetectable by the time the rash appeared. Given the timing of the reaction, involvement of heparin or alteplase is unlikely.

With a score of 7 out of a possible 13 points on the Naranjo Adverse Drug Reaction Probability scale, causality between rivaroxaban and the clinical events observed was deemed probable. ${ }^{28}$ In addition, the causative effect of rivaroxaban can be established with more certainty in this case than in similar cases reported previously, because the patient had not taken any other medications. Furthermore, the results of skin biopsy supported this diagnosis. Histopathological examination of the skin biopsy sample showed spongiotic dermatitis with perivascular lymphocytes and eosinophilic infiltrates, which are frequently present in cases of drug-induced skin eruption. ${ }^{29}$ It is also important to note that following rivaroxaban cessation, there was continual improvement until complete resolution of the rash and hepatic cytolysis.

\section{CONCLUSION}

In this report, we have described a case of DIHS to rivaroxaban manifested by a mild pruritic papular rash and marked elevation of liver enzymes without eosinophilia. Clinicians should be aware of the possibility of DIHS with rivaroxaban, because early discontinuation of the offending drug is mandatory.

\section{References}

1. Samama MM. The mechanism of action of rivaroxaban - an oral, direct factor Xa inhibitor-compared with other anticoagulants. Thromb Res. 2011;127(6): 497-504.

2. Turpie AGG. Oral, direct factor Xa inhibitors in development for the prevention and treatment of thromboembolic diseases. Arterioscler Thromb Vasc Biol. 2007;27(6):1238-47.

3. Yeh CH, Hogg, K, Weitz JI. Overview of the new oral anticoagulants: opportunities and challenges. Arterioscler Thromb Vasc Biol. 2015;35(5):1056-65.

4. Xarelto (rivaroxaban) [package insert]. Ontario (CA): Bayer Inc; 2015.

5. Yeh CH, Fredenburg JC, Weitz JI. Oral direct factor Xa inhibitors. Circ Res. 2012;111(8):1069-78

6. Vemuri C, Salehi P, Bernarroch-Gampel J, McLaughlin LN, Thompson RW. Diagnosis and treatment of effort-induced thrombosis of the axillary subclavian vein due to venous thoracic outlet syndrome. J Vasc Surg Venous Lymphat Disord. 2016;4(4):485-500.

7. Alla VM, Natarajan N, Kaushik M, Warrier R, Nair CK. Paget-Schroetter syndrome: review of pathogenesis and treatment of effort thrombosis. West $\mathrm{J}$ Emerg Med. 2010;11(4):358-62.

8. Pichler WJ, Naisbitt DJ, Park BK. Immune pathomechanism of drug hypersensitivity reactions. J Allergy Clin Immunol. 2011;127(3 Suppl):S74-81.

9. Yawalkar N. Drug-induced exanthems. Toxicology. 2005;209(2):131-4.

10. Schrijvers R, Gilissen L, Chiriac AM, Demoly P. Pathogenesis and diagnosis of delayed-type drug hypersensitivity reactions, from bedside to bench and back. Clin Transl Allergy. 2015;5:31.

11. Kuljanac I. Mechanisms of drug hypersensitivity reactions and the skin. Recent Pat Inflamm Allergy Drug Discov. 2008;2(1):64-71.

12. Hausmann O, Schnyder B, Pichler WJ. Drug hypersensitivity reactions involving skin. In: Uetrecht J, editor. Adverse drug reactions (Handb Exp Pharmacol. 196). Berlin (Germany): Springer; 2010. p. 29-55.

13. Raschi E, Poluzzi E, Koci A, Salvo F, Pariente A, Biselli M, et al. Liver injury with novel oral anticoagulants: assessing post-marketing reports in the US Food and Drug Administration adverse event reporting system. Br J Clin Pharmacol. 2015;80(2):285-93.

14. Prasannan N, Soultati A, Harrison C, Yeghen T, Radia D. 2 cases of idiopathic hypereosinophilic syndrome: a rare disorder with severe thrombohaemorrhagic complications [abstract]. Haematologica. 2013;98 Suppl 1:627. 
15. Chaaya G, Jaller-Char J, Ghaffar E, Castiglioni A. Rivaroxaban-induced leukocytoclastic vasculitis: a challenging rash. Ann Allergy Asthma Immunol. 2016;116(6):577-8.

16. Altin C, Ozturkeri OAY, Gezmis E, Askin U. Angioedema due to the new oral anticoagulant rivaroxaban. Ann Card Anaesth. 2014;17(2):173-4.

17. Vernon HM, Nielsen AK, O'Bryan EC. Hypersensitivity reaction after administration of rivaroxaban (Xarelto). Am J Emerg Med. 2016;34(7):1325.e1-2.

18. Yates J, Choudhry M, Keys G. A case report describing a suspected rivaroxaban hypersensitivity reaction in a surgical patient. J Clin Pharm Ther. 2013;38(2): 159-61.

19. Barrett P, Vuppalanchi R, Masuoka H, Chalasani N. Severe drug-induced skin and liver injury from rivaroxaban. Dig Dis Sci. 2015;60(6):1856-8.

20. Radu C, Barnig C, de Blay F. Rivaroxaban-induced drug reaction with eosinophilia and systemic symptoms. J Investig Allergol Clin Immunol. 2016; 26(2):124-6.

21. Husain Z, Reddy BY, Schwartz RA. DRESS syndrome: Part II. Management and therapeutics. J Am Acad Dermatol. 2013;68(5):709.e1-e9.

22. Snyder DJ, Matusik FB. Rivaroxban-induced serum sickness after total knee arthroplasty. Am J Health Syst Pharm. 2015;72(18):1567-71.

23. Hill MD, Barber PA, Takahashi J, Demchuk AM, Feasby TE, Buchan AM. Anaphylactoid reactions and angioedema during alteplase treatment of acute ischemic stroke. CMAJ. 2000;16(9)2:1281-4.

24. Linnik W, Tintinalli JE, Ramos R. Associated reactions during and immediately after rtPA infusion. Ann Emerg Med. 1989;18(3):234-9.

25. Molinaro G, Gervais N, Adam A. Biochemical basis of angioedema associated with recombinant tissue plasminogen activator treatment: an in vitro experimental approach. Stroke. 2002;33(6):1712-6.

26. Tanswell P, Tebbe U, Neuhaus KL, Gläsle-Schwarz L, Wojcik J, Seifried E. Pharmacokinetics and fibrin specificity of alteplase during accelerated infusions in acute myocardial infarction. J Am Coll Cardiol. 1992;19(5):1071-5.

27. Gonzalez-Delgado P, Fernandez J. Hypersensitivity reactions to heparins. Curr Opin Allergy Clin Immunol. 2016;16(4):315-22.

28. Naranjo CA, Busto U, Sellers EM, Sandor P, Ruiz I, Roberts EA, et al. A method for estimating the probability of adverse drug reactions. Clin Pharmacol Ther. 1981;30(2):239-45.

29. Weyers W, Metze D. Histopathology of drug eruptions-general criteria, common patterns, and differential diagnosis. Dermatol Pract Concept. 2011;1(1):33-47.
Charles-Olivier Chiasson, PharmD, MSc, was, at the time this manuscript was originally prepared, a Pharmacy Resident at the McGill University Health Centre and a student in the Faculty of Pharmacy, Université de Montreal, Montréal, Quebec. He is now a Pharmacist at the Centre hospitalier universitaire Sainte-Justine, Montréal, Quebec.

Arnaud Canneva, PharmD, MSc, was, at the time this manuscript was originally prepared, a Pharmacy Resident at the McGill University Health Centre and a student in the Faculty of Pharmacy, Université de Montreal, Montréal, Quebec. He is now a Pharmacist with the Montreal Heart Institute, Montréal, Quebec.

François-Olivier Roy, PharmD, MSc, was, at the time this manuscript was originally prepared, a Pharmacy Resident at the Hôpital du Sacré-Coeur de Montréal and a student in the Faculty of Pharmacy, Université de Montreal, Montréal, Quebec. He is now a Pharmacist at Hotel-Dieu d'Arthabaska, Victoriaville, Quebec.

Maxime Doré, BSc, BPharm, MSc, is a Pharmacist at the Hôpital du SacréCoeur de Montréal, Montréal, Quebec.

Charles-Olivier Chiasson, Arnaud Canneva, and François-Olivier Roy contributed equally to this manuscript.

Competing interests: None declared.

Address correspondence to:

Dr Charles-Olivier Chiasson

Pharmacy Department

Centre hospitalier universitaire Sainte-Justine

3175, chemin de la Côte-Sainte-Catherine

Montréal QC H3T 1C5

e-mail: charles-olivier.chiasson.hsj@ssss.gouv.qc.ca

Funding: None received.

\section{CORRECTION}

\section{Should Patients Continue to Receive Statins Once They Reach 80 Years of Age? The "Con" Side: Correction}

In the "Con" side of the recent Point Counterpoint debate about use of statins in patients 80 years of age and older, ${ }^{1}$ one of the values for number needed to treat (NNT), based on data in a previously published article, ${ }^{2}$ was incorrect. In paragraph 3 of the section "Benefits and Likelihood of 'Success' May Be Exaggerated, While Harms Are Underestimated", the NNT for secondary prevention of myocardial infarction with statins in older patients should be 38 , not 83 . The complete, corrected sentence reads as follows, with the corrected NNT shown in bold.

For secondary prevention with statins in older patients (mean age 69 years), the most optimistic assessment of the evidence suggests an absolute risk reduction of $2.6 \%$ for myocardial infarction (NNT 38), 1.7\% for stroke (NNT 58), and 3.8\% for all-cause mortality (NNT 28) over 3.5 years. ${ }^{2}$
References

1. Tejani AM, O'Sullivan C. Should patients continue to receive statins once they reach 80 years of age? The "con" side. Can J Hosp Pharm. 2017;71(3):244-6.

2. Afilalo J, Duque G, Steele R, Jukema JW, de Craen AJ, Eisenberg MJ. Statins for secondary prevention in elderly patients a hierarchical Bayesian meta-analysis. J Am Coll Cardiol. 2008;51(1):37-45. 